\title{
Fundamentals of the Treatment of Male Erectile Dysfunctions with Low Intensity Shockwaves
}

\author{
César Eisner ${ }^{1}$, Mauricio Salas ${ }^{2}$, Daniel Moya ${ }^{3}$
}

\section{Abstract}

Male erectile dysfunction (ED) is one of the most common problems among men worldwide. No single diagnostic method evaluates all the variables of this complex condition. To achieve good therapeutic results, it is essential to base the treatment on an accurate diagnosis. Hemodynamic exploration by echo Doppler of the cavernous arteries, especially since the incorporation of intracavernous administration of vasoactive drugs, is a useful tool that allows the evaluation of erectile dysfunction in the arterial phase. It is also considered to be the choice in the assessment of the corporoveno-occlusive mechanism. Different treatment methods are used, being PDE5 (sildenafil and tadalafil), the treatment of the first choice in several conditions. The number of publications of low-intensity extracorporeal shockwave treatment (LIESWT) for ED has increased dramatically in recent years. Scientific evidence regarding the application of LI-ESWT for the treatment of erectile dysfunction is still controversial. Inclusion criteria of the studies and the wide variety of treatment protocols have been criticized. On the other hand, most of these studies report encouraging results with no short-term adverse effects, regardless of variation in LI-ESWT setup parameters or treatment protocols.

Keywords: Erectile dysfunction, Linear shock wave, Linear shock wave therapy, Shear wave elastography

\section{Introduction}

Since the beginning of the 21 st century, low intensity focused shock wave treatments (LIESWT) have been applied to male erectile dysfunctions (MED) with good results in clinical practice $[1,2,3,4,5]$. Nevertheless, their acceptance by the medical community did not have an absolute consensus due to the initial lack of knowledge of the scientific background that support their use and the complexity of methodological analysis, considering the wide variety patients of characteristics, and the use of different devices and protocols.

Fortunately, the continuous and immense contribution of researchers, the development of new diagnostic technologies and the activities carried out by the ISMST (International Society for Medical Shockwave Treatment) [6] and Onlat (IberoLatinamerican Shockwave and Tissue Engineering Federation) [7] allow us today to deepen our understanding of the biological mechanisms that underpin the results obtained with this type of treatment.

The purpose of this review is to provide an overview about the use of low intensity focused shock wave treatments in Erectile Dysfunction (ED) and discuss the role of ultrasound in the diagnosis and follow-up of this condition.

\section{Erectile Dysfunction}

$\mathrm{ED}$ is one of the most common problems among men worldwide [8]. No single diagnostic method evaluates all the variables of this complex condition. The first thing to consider is the psychosocial context of the patient. Biological factors such as hormonal disorders (testosterone), metabolic disorders (blood glucose, cholesterol), and even drug iatrogenesis must be excluded.

To achieve a penile erection an erogenous stimulus is required, which by parasympathetic way causes the release in corpora cavernosa (CC) of nitric oxide with vasodilator action, which in turn promotes the action of guanosin-3', 5'-cyclic monophosphate (cGMP), with muscle relaxant effect [9]. In this way, the helicine veins of the $\mathrm{CC}$ will be filled with blood and expand, producing penile tumescence. This ability to expand is known as "compliance."

In addition, a correct veno-occlusive mechanism will be necessary to contain the blood within the vessels long enough to obtain a satisfactory erection and sexual intercourse. This containment is fundamentally mediated by the tone of the tunica albuginea and the state of the veins of the penis. ED can be caused if there are alterations in the mechanisms of vascular dilation, muscle relaxation or the capacity to contain the tunica albuginea, among others.

The loss of elasticity, or in other words, the increase in the rigidity of the $\mathrm{CC}$, which in many cases increases with age or due to comorbidities present from the age of 50 [10], limits compliance and is therefore the

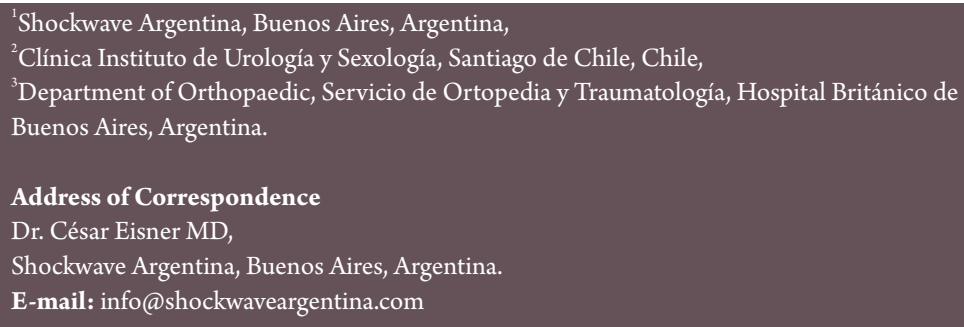

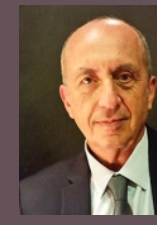

Dr. César Eisner

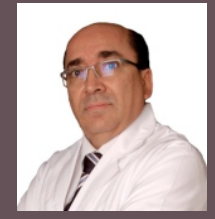

Dr. Mauricio Salas

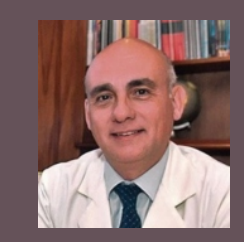

Dr. Daniel Moya

Submitted Date: 25 October 2021, Review Date:20 November 2021, Accepted Date: 12 December 2021 \& Published: 31 December 2021

(C) 2021 by Journal of Regenerative Science | Available on www.jrsonweb.com | DOI:10.13107/jrs.2021.v01.i01.015

This is an open access journal, and articles are distributed under the terms of the Creative Commons Attribution-NonCommercial-ShareAlike 4.0 License (https://creativecommons.org/licenses/by-nc-sa/4.0/), which allows others to remix, tweak, and build upon the work non-commercially, as long as appropriate credit is given and the new creations are licensed under the identical terms. 
Role of Ultrasound in the Diagnosis and Follow-up of Cases of ED

Hemodynamic exploration by echo-Doppler of the cavernous arteries, especially since the incorporation of intracavernous administration of vasoactive drugs, is a useful tool that allows the evaluation of ED in the arterial phase. It is also considered to be the choice in the assessment of the corporovenoocclusive mechanism (or venous phase), and it has gradually displaced tests such as cavernosography and dynamic cavernosometry.

For the monitoring and evaluation of therapeutic results, there are many tools that, when combined, offer important information. Self-reported erectile symptoms, the International Index of Erectile Function-5 and Erection Hardness Scores, nocturnal penile tumescence and rigidity and cavernous duplex Doppler ultrasound are very useful options [11].

Therapeutic results can be evaluated not only by clinical response but also with changes in ultrasound images. Color Doppler ultrasound and a qualitative-quantitative shear elastography (2D SWE) of the penis are effective to verify the presence of vascular dilatations or an increase in the rigidity of the CC.

Color Doppler ultrasound can put in evidence vascular inability to contribute to an efficient veno-occlusive mechanism, but the results of Doppler US should be interpreted with care by the clinicians due to the sympathetic overtone and underlying psychological disturbances in some patients. On the other hand, the 2D SWE can qualify and quantitatively show the degree of elasticity of the CC.

The combined use of color Doppler cause ofED.

ultrasound in conjunction with 2D-SWE (shear wave elastography), allows not only a quali-quantitative and non-operator dependent diagnosis of the vasculofibrogenic ED and De Peyronie disease, but also monitor therapeutic results after LIESWT (Fig. 1).

\section{ED Treatment}

To achieve good therapeutic results in ED, it is essential to base the treatment on an accurate diagnosis, since not all ED should be treated with LI-ESWT [12]. A comprehensive biopsychosocial evaluation and treatment should be considered in the first instance, involving psychological and biological aspects. Considering that ED is multicausal, an in-depth evaluation must be appealed to rule out factors such as medications whose collateral effects affect erectile function (beta-blockers, antiandrogens, some antidepressants, etc.), psychogenic factors, neurological disorders, endocrinological factors, etc. [11]. Excluding these problems, -PDE5 (sildenafil, tadalafil) constitutes the treatment of first choice in ED [11]. It's effect allows, after erogenous stimulation, firm and sustained erections since it maintain high levels of cGMP. However, if the CC show increased stiffness (visible with 2D SWE), whether due to fibrosis, increased collagen fibers, or decreased muscle fibers, adequate compliance will not be possible, and therapeutic efficacy of I-PDE5 will be reduced.

Patients with a "vascular-fibrogenic" ED, diagnosed by color Doppler or 2D SWE, are suitable for a successful LI-ESWT, regardless of the presence of other etiopathogenic factors, which simultaneously and in parallel will require specific treatment.

Shockwaves can also have a positive effect on

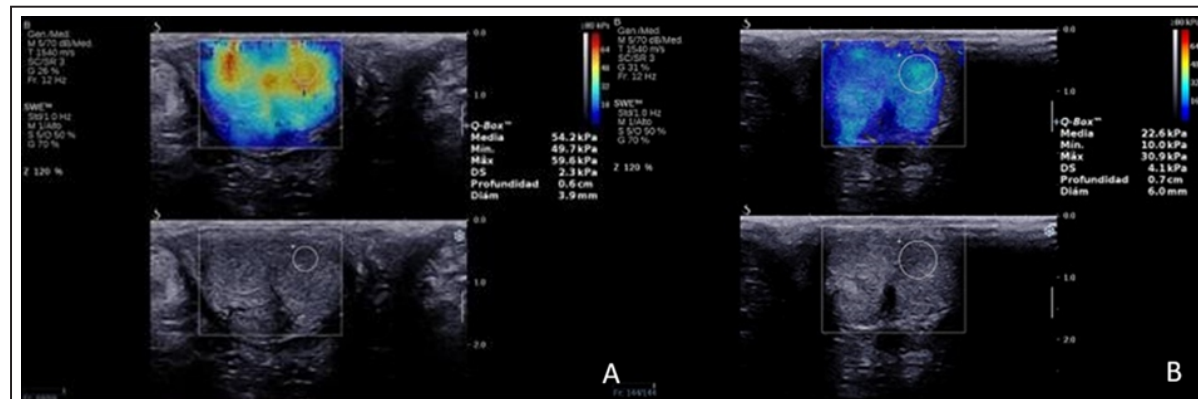

Figure 1: (a) Shear wave elastography (2D-SWE) of the cavernous bodies (CC) pre-treatment. Increased central stiffness is seen in both CC and in the albugineal septum. Average elasticity (average E.): $54.2 \mathrm{kPa}$. (b) Post-treatment shear wave elastography (2D$\mathrm{SWE}$ ) of the corpora cavernosa (CC). Normal stiffness is seen in both CC and tunica albuginea. Average E. 22.6 kPa. (Normal value: $<25 \mathrm{kPa}$.) "psychogenic" -sympathetic/adrenergic ED $[1,13]$. Alpha-2 adrenergic receptors modulate/inhibit the sympathetic tone making the onset of erections easier. By changing the ratio of alpha-1/alpha- 2 adrenergic receptors in favor of alpha-2 adrenergic receptors, ESWT may be able to decrease the sympathetic activity within the cavernous bodies and finally may facilitate erection in the so-called psychogenic ED patients with an increased sympathetic tone $[1,13]$.

Another cause of ineffectiveness may be due to the loss of elastic capacity of the tunica albuginea, causing diffuse venous leakage by not being able to efficiently occlude the subalbugineal vessels. The increased diffuse stiffness of the CC, in addition to ED, can cause retraction of the penis, and in cases of localized stiffness, with or without fibrous or calcified plaques, produce Peyronie's disease. For these reasons, reducing the rigidity of $\mathrm{CCs}$ to treat vasculo-fibrogenic ED, penile retraction or penile curvature is one of the objectives proposed to be achieved with LIESWT.

In cases of venous leakage, the ability of LIESWT to revascularize smooth muscle will make it possible to increase its tone and trophism, indirectly and extrinsically contributing to the reduction of the caliber of the venous dilatations observed.

Liu et al. [14] described in detail the specific signaling pathways involved in penile CC: Wnt, Focal Adhesion Kinase, Extracellularsignal-regulated kinase (ERK) on membranes and in the reticuloendothelial system, ATP and Brain-derived neurotrophic factor (BDNF) on organelles, obtaining transcription of specific genes with regenerative effects on muscle and nerve fibers, as well as regulators of the levels of intra and extracellular $\mathrm{Ca}$, essential to maintain the tone of the endothelial smooth muscle in flaccidity or its relaxation in erection.

In recent years, notable scientific papers have been published that account for the biomolecular and genetic mechanisms responsible for the improvement observed in ED-LI ESWTs. ESWT acts through mechanotransduction, which is the mechanism by which a shock wave generates a biological response $[15,16]$. Biological responses, the result of stimulating cell nuclei, consist of the production and release 
of growth factors and structural proteins.

In 2003, Wang [17] published the first molecular biology study showing that, through selective and specific signaling pathways, LI-ESWT activates cell nuclei to produce the transcription of genes that allow a repairing or restorative response to be given to a specific stimulus, generating muscle fibers. Biological responses, the product of stimulating cell nuclei, consist of the production and release of growth factors and structural proteins.

LI-ESWT can regenerate peripheral nerves from improving functional results [18], from the production of neurotrophic factors (Nerve Growth Factor, BDNF, Neurotrophin 3, FCN: Ciliary Factor neurotrophic, etc.) and to activate Schwann cells and the signaling pathways that promote cell proliferation and activation (ERK 1/2, MAPK, P75 PERK/ATF4, SER and BDNF), being particularly effective in the treatment of Neurogenic ED Post-Radical Prostatectomy (DENPPR.) With nerve sparing.

Specific contraindications to LI-ESWT use must be consider, such as the use of pacemakers, arrhythmias, coagulation disorders and anticoagulated patients [6]. Local infections and tumors or a prosthesis in the penis are also contraindications.

\section{Scientific evidence}

Scientific evidence regarding the application of LI-ESWT for the treatment of ED is still controversial $[19,20]$, but most of the experimental and clinical studies reported positives results $[2,4,19,21,22,23,24,25$, $26,27]$.

Although widely offered in numerous centers, LI-SWT is not or FDA or Health Canada -approved $[20,28]$ for clinical use for
ED. The American Urological Association states "For men with ED, low-intensity extracorporeal shock wave therapy (ESWT) should be considered investigational. (Conditional Recommendation; Evidence Level: Grade C) [29].

The British Society for Sexual Medicine Guidelines on the Management of ED in Men recommends: "Recent meta-analyses suggest that there might be a place for low-intensity extracorporeal shock wave treatment in men with mild ED wishing to avoid medical therapy and as a salvage therapy for PDE5I this might be a preferred option for patients failing oral therapy but reluctant to move to injection therapy" [30].

The guidelines of the European Association of Urology propose a treatment algorithm in which they state: "The use of low-intensity extracorporeal shockwave therapy (LISWT) has been increasingly proposed as a treatment for ED over the last decade. Overall, most of these studies reported encouraging results, regardless of variation in LI-ESWT set-up parameters or treatment protocols. However, the publication of unequivocal evidence from additional RCTs and longer-term follow-up would provide more confidence regarding the use of LISWT (including detailed number of pulses per patient, treatment protocols) for ED patients. Therefore, clear and definitive recommendations cannot be given" [31].

According to the Canadian Urological Association studies have different treatment protocols (shockwave devices, energy levels, applied pulses, and schedule of treatments), various sham treatments, inconsistent followup timing, short follow-up, and varying metrics, resulting in significant heterogeneity between the studies [28]. While this is true, most studies report good results.

Porst [1] reported in a review published in 2020 that clinical studies with six different devices, in patients with organic ED, although using different protocols, in all cases obtained positive results. Three metaanalyses have reported significant benefit to Li-ESWT in the treatment of ED $[2,4,19]$.

Nor have any significant complications been reported with the use of Li-ESWT, being an outpatient procedure that does not require anesthesia for these conditions because it is well tolerated.

The protocol used by the first author of this review includes the application of up to 6 sessions of LI-ESWT, at a rate of 3000 waves per session, 1-2 times a week, with a frequency of 3-4 Hz and with an energy of $0.25 \mathrm{mJoules} / \mathrm{mm} 2$.

It has been possible to reduce the stiffness values of the CC between 5 and $15 \mathrm{kPa}$ per session. Results obtained in the ultrasound studies showed a direct correlation with the clinical improvement, either with low dose or without the use of I-PDE5, given the lower tissue resistance to vasodilation.

\section{Conclusion}

Scientific evidence regarding the application of Li-ESWT for the treatment of ED is still controversial. The number of publications of LI-ESWT for ED has increased dramatically in recent years, but the inclusion criteria of the studies and the wide variety of treatment protocols have been criticized. Most of these studies presented encouraging results with no short-term adverse effects, regardless of variation in LI-ESWT setup parameters or treatment protocols.

Declaration of patient consent: The authors certify that they have obtained all appropriate patient consent forms. In the form, the patient has given his consent for his images and other clinical information to be reported in the Journal. The patient understands that his name and initials will not be published, and due efforts will be made to conceal his identity, but anonymity cannot be guaranteed.

Conflicts of Interest: Nil. Source of Support: None.

\section{References}

1. Porst H. Review of the current status of low intensity extracorporeal shockwave therapy (Li-ESWT) in erectile dysfunction (ED), Peyronie's disease (PD), and sexual rehabilitation after radical prostatectomy with special focus on technical aspects of the different marketed ESWT devices including personal experiences in 350 patients. Sex Med Rev 2021;9:93122.

2. Clavijo RI, Kohn TP, Kohn JR, Ramasamy R. Effects of low-intensity extracorporeal shockwave therapy on erectile dysfunction: A systematic review and meta-analysis. J Sex Med 2017;14:27-35.
3. Dong L, Chang D, Zhang X, Li J, Yang F, Tan K, et al. Effect of lowintensity extracorporeal shock wave on the treatment of erectile dysfunction: A systematic review and meta-analysis. Am J Mens Health 2019;13:1557988319846749.

4. Lu Z, Lin G, Reed-Maldonado A, Wang C, Lee YC, Lue TF. Low-intensity extracorporeal shock wave treatment improves erectile function: $A$ systematic review and meta-analysis. Eur Urol 2017;71:223-33.

5. Mo DS, Zhan XX, Shi HW, Cai HC, Meng J, Zhao J, et al. Efficacy and safety of low-intensity extracorporeal shock wave therapy in the treatment 
of ED: A meta-analysis of randomized controlled trials. Zhonghua Nan Ke Xue 2019;25:257-64.

6. Consensus Statement on ESWT Indications and Contraindications. A $v$ a i l a b le f $r \circ \mathrm{m}$ : https://www.shockwavetherapy.org/fileadmin/user_upload/dokumente/P DFs/Formulare/ISMST consensus statement on indications and cont raindications_20161012_final.pdf [Last accessed on 2021 Oct 11].

7. Ondas de Choque en Medicina: La Nueva Frontera. Available from: https://onlat.net/?page_id=2491 [Last accessed on 2021 Oct 11].

8. Kessler A, Sollie S, Challacombe B, Briggs K, Van Hemelrijck M. The global prevalence of erectile dysfunction: A review. BJU Int 2019. Doi: 10.1111/bju. 14813 Epub Ahead of Print.

9. Melman A, Rehman J. Pathophysiology of erectile dysfunction. Mol Urol 1999;3:87-102.

10. Jønler M, Moon T, Brannan W, Stone NN, Heisey D, Bruskewitz RC. The effect of age, ethnicity and geographical location on impotence and quality of life. Br J Urol 1995;75:651-5.

11. Huang SA, Lie JD. Phosphodiesterase-5 (PDE5) inhibitors in the management of erectile dysfunction. PT 2013;38:407-19.

12. Krzastek SC, Bopp J, Smith RP, Kovac JR. Recent advances in the understanding and management of erectile dysfunction. F1000Res. 2019;8:F1000 Faculty Rev-102.

13. Sokolakis I, Dimitriadis F, Psalla D, Karakiulakis G, Kalyvianakis D, Hatzichristou D. Effects of low-intensity shock wave therapy (LiST) on the erectile tissue of naturally aged rats. Int J Impot Res 2019;31:162-9.

14. Liu T, Shindel AW, Lin G, Lue TF. Cellular signaling pathways modulated by low-intensity extracorporeal shock wave therapy. Int J Impot Res 2019;31:170-6.

15. d'Agostino MC, Craig K, Tibalt E, Respizzi S. Shock wave as biological therapeutic tool: From mechanical stimulation to recovery and healing, through mechanotransduction. Int J Surg 2015;24 Pt B:147-53.

16. Moya D, Ramón S, Schaden W, Wang CJ, GuiloffL, Cheng JH. The role of extracorporeal shockwave treatment in musculoskeletal disorders. $J$ Bone Joint Surg 2018;100:251-63.

17. Wang CJ. An overview of shock wave therapy in musculoskeletal disorders. Chang Gung Med J 2003;26:220-32.

18. Qiu X, Lin G, Xin Z, Ferretti L, Zhang H, Lue TF, et al. Effects of lowenergy shockwave therapy on the erectile function and tissue of a diabetic rat model. J Sex Med 2013;10:738-46.

19. Rizk PJ, Krieger JR, Kohn TP, PastuszakAW. Low-intensity shockwave therapy for erectile dysfunction. Sex Med Rev 2018;6:624-30.
20. Katz JE, Clavijo RI, Rizk P, Ramasamy R. The basic physics of waves, soundwaves, and shockwaves for erectile dysfunction. Sex Med Rev 2020;8:100-5.

21. Yee CH, Chan ES, Hou SS, Ng CF. Extracorporeal shockwave therapy in the treatment of erectile dysfunction: A prospective, randomized, doubleblinded, placebo controlled study. Int J Urol 2014;21:1041-5.

22. Kalyvianakis $D$, Hatzichristou $D$. Low-intensity shockwave therapy improves hemodynamic parameters in patients with vasculogenic erectile dysfunction: A triplex ultrasonography-based sham-controlled trial. J Sex Med 2017;14:891-7.

23. Fojecki GL, Tiessen S, Osther PJ. Effect of low-energy linear shockwave therapy on erectile dysfunction-a double-blinded, shamcontrolled, randomized clinical trial. J Sex Med 2017;14:106-12.

24. Olsen AB, Persiani M, Boie S, Hanna M, Lund L. Can low-intensity extracorporeal shockwave therapy improve erectile dysfunction? A prospective, randomized, double-blind, placebo-controlled study. Scand J Urol 2015:49:329-33.

25. Vardi Y, Appel B, Kilchevsky A, Gruenwald I. Does low intensity extracorporeal shock wave therapy have a physiological effect on erectile function? Short-term results of a randomized, double-blind, sham controlled study. J Urol 2012;187:1769-75.

26. Pelayo-Nieto M, Linden-Castro E, Alias-Melgar A, Espinosa-Pérez Grovas D, Carreño-de la Rosa F, Bertrand-Noriega F, et al. Linear shock wave therapy in the treatment of erectile dysfunction. Actas Urol Esp 2015;39:456-9.

27. Huang YP, Liu W, Liu YD, Zhang M, Xu SR, Lu MJ. Effect of lowintensity extracorporeal shockwave therapy on nocturnal penile tumescence and rigidity and penile haemodynamics. Andrologia 2020;52:e13745

28. Domes T, Najafabadi BT, Roberts M, Campbell J, Flannigan R, Bach P, et al. Canadian urological association guideline: Erectile dysfunction. Can Urol Assoc J 2021;15:310-22.

29. Burnett AL, Nehra A, Breau RH, Culkin DJ, Faraday MM, Hakim LS, et al. Erectile dysfunction: AUA guideline. JUrol 2018;200:633-41.

30. Hackett G, Kirby M, Wylie K, Heald A, Ossei-Gerning N, Edwards D, et al. British society for sexual medicine guidelines on the management of erectile dysfunction in men-2017. J Sex Med 2018;15:430-57.

31. Hatzimouratidis K, Giuliano F, Moncada I, Muneer A, Salonia A, Verze P. EAU Guidelines on Erectile Dysfunction, Premature Ejaculation, Penile Curvature and Priapism. European Association of Urology; 2019. p. 23. Available from: https://uroweb.org/wp-content/uploads/EAU-guidelineson-male-sexual-dysfunction-2019.pdf [Last accessed on 2021 Oct 11].

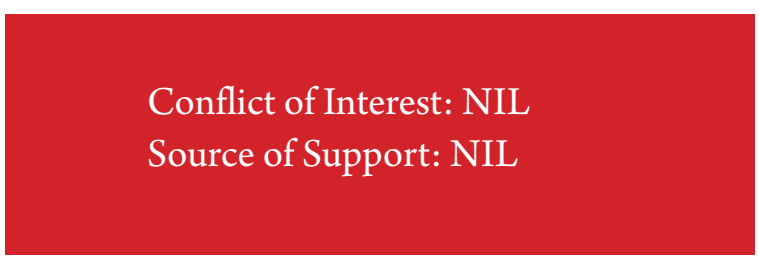

\section{How to Cite this Article}

Eisner C, Salas M, Moya D | Fundamentals of the Treatment of Male Erectile Dysfunctions with Low Intensity Shockwaves | Journal of Regenerative Science December 2021; 1(1):26-29. 